\title{
营养基因组学的研究进展
}

陈琴，王文君，上官新晨，徐明生

江西农业大学食品科学与工程学院, 南昌 330045

摘要: 营养学是一门古老的学科, 为人们的健康保护和疾病预防提供了重要的理论指导。随着分子生物学技术 的发展, 它已成为 21 世纪生命科学研究最为主要的技术之一。分子生物学与营养学的结合, 产生了分子营养 学。而基因组学与营养学的结合, 则形成了营养基因组学。营养基因组学涵盖了一个广泛的领域, 它研究营养 素和基因表达的相互影响, 预测其对营养素的反应。基因组学技术可以帮助确认一些与疾病发生有关的基因, 人们可以根据各自的基因图谱制定一份个性化的饮食方案, 以此防病治病, 使人们的健康状况通过调整饮食来 达到最佳。文中重点介绍了营养基因组学的主要研究方法及其应用。

关键词: 个人营养食谱; 基因组学; 单核苷酸多态; 营养基因组学

\section{Research progresses on nutrigenomics}

\author{
CHEN Qin, WANG Wen-Jun, SHANGGUAN Xin-Chen, XU Ming-Sheng \\ College of Food Science and Engineering, Jiangxi Agricultural University, Nanchang 330045, China
}

\begin{abstract}
Nutrition science is an age-old subject, and offers important theoretic instructions for human health protection and disease prevention. With the development of molecular biology, it will be a key technique in the 21 century. Combination of molecular biology and nutrition, bio-nutrition is formed. While the combination of genome and nutrition, nutrigenomics is developed. Nutrigenomics covers a wide range of areas, which studies the interactive effects between nutrients and genes expression, and predicts the expressive response to the nutrients. Genomics technology can help us to identify some disease-related genes, thereby people establish individual diet and make their heath attained the best status through adjusting diet. This paper focuses on the main research technology of nutrigenomics and its application.
\end{abstract}

Keywords: individual nutritional diet; genomics; SNP; nutrigenomics

营养学是研究人体营养规律及其改善措施的科 学。人们在很早以前就开始了营养学的研究, 如我 国的医学古籍《黄帝内经·素问》中就提出了“五谷为 养、五果为助、五畜为益、五菜为充”等朴素的合理 营养概念。而西方的医学始祖希波克拉底在公元前 400 年前也提出了食品中的特殊成分对于维持生命 是必不可少的。但真正意义上的营养学诞生却是在
发现了构成人体重要物质的 18 世纪后期, 从 1900 年至今, 营养学研究不断深入, 已经历了 3 个阶段。 二战后, 营养学进入了基于实验科学技术的鼎盛时 期。20 世纪后半叶, 人类进入了细胞时代, 主要研 究营养素在体内代谢、生理功能及其对组织细胞的 影响。而分子生物学划时代的到来, 为营养学向微 观世界发展、探索生命奥秘提供了理论基础。特别

收稿日期: 2007-08-10; 修回日期: 2007-11-30

基金项目: 江西农业大学博士启动基金(编号 :2004)和江西农业大学自然科学基金(编号 :2228)[Supported by Program of Doctoral Initiation of Jiangxi Agricultural University(No. 2004) and Program of Natural Science Fund of Jiangxi Agricultural University(No. 2228)] 作者简介: 陈琴(1984-), 女, 江西新余人, 硕士研究生, 研究方向 : 营养基因组学。E-mail: chenqin19840123@126.com 通讯作者:王文君(1971-), 男, 江西玉山人, 博士, 副教授, 研究方向 : 生物化学与分子生物学。E-mail: wwjun9999@sina.com 
是人类及模式生物的基因组草图、基因组序列图相 继绘制完成，为人类阐明基因组及所有基因的结构 与功能，揭开生命奥秘奠定了基础。营养科学也由 营养素对单个基因表达及其作用的分析，开始朝着 基因组及其表达产物在代谢调节中的作用方向发 展。在此背景下, 营养基因组学(Nutritional genomics, 有时也称为 Nutrigenomics) 应运而生, 并迅速成为营 养学研究的新前沿。2002 年 2 月和 2003 年 11 月, 在 荷兰先后召开了第一届和第二届国际营养基因组会 议, 凸现了营养基因组学研究的重要性。

\section{1 营养基因组学的发展及概念}

1953 年, Watson和Crick ${ }^{[11}$ 发现了被称为“生命奥 秘”的DNA结构, DNA结构解释了遗传物质是如何复 制和传递信息的。DNA这种优雅神秘的双螺旋结构 的发现, 引发的革命震动了生物学界和医学界, 标 志着分子生物学的开始。1961 年, DNA中碱基对序 列转录基因密码的破译成功，标志着基因时代的到 来。以人类基因组 “工作框架图”完成为标志 ${ }^{[2]}$, 生 命科学已进入了后基因组时代 ${ }^{[3]}$ 。美国科学家 Thomas Roderick(1986)提出了基因组学(Genomics), 主要内容包括以全基因组测序为目标的结构基因组 学(Structural genomics)和以基因功能鉴定为目标的 功能基因组学(Functional genomics) ${ }^{[4]}$ 。

随着基因组学研究的发展以及人类基因组计划 的实施和完成, 科学界普遍认为, 这种让人困惑的 现象很可能都是由个体间的基因差异造成的。借助 各种不断发展的先进研究手段, 已有不少科学家开 始从理论和实践两方面更深入地认识基因与饮食间 的相互影响, 营养学研究也由此迈入了一个崭新的 时代一一基因时代”，一门以专门研究人的饮食与 其自身基因之间交互作用为目标的营养学研究领域 内的新兴学科—营养基因组学也逐渐引起人们的 兴趣 ${ }^{[5]}$ 。营养基因组(Nutrigenomics)是 2000 年提出 的一种新的营养学理论, 是继药物之后源于人类基 因组计划的个体化治疗的第二次浪潮。营养基因组 学所涉及的学科有营养学、分子生物学、基因组学、 生物化学、生物信息学等, 从这个层面上看, 营养基 因组学是基于多学科的边缘学科。

营养基因组学是高通量基因组技术在日粮营养 素与基因组相互作用及其与健康关系研究中的应 用 ${ }^{[6]}$ 。营养基因组学是研究营养素和食物化学物质 在人体中的分子生物学过程以及产生的效应, 对人
体基因的转录、翻译表达以及代谢机制，其可能的 应用范围包括营养素作用的分子机制、营养素的人 体需要量、个体食谱的制定以及食品安全等, 它强 调对个体的作用 ${ }^{[7]}$ 。营养基因组学研究首先要解决 的两个问题是：建立一个具有很高预测价值的膳食 推荐量用以预防疾病、降低不可估计后果的风险; 设计有效的膳食用以控制慢性疾病 ${ }^{[8]}$ (图 1)。

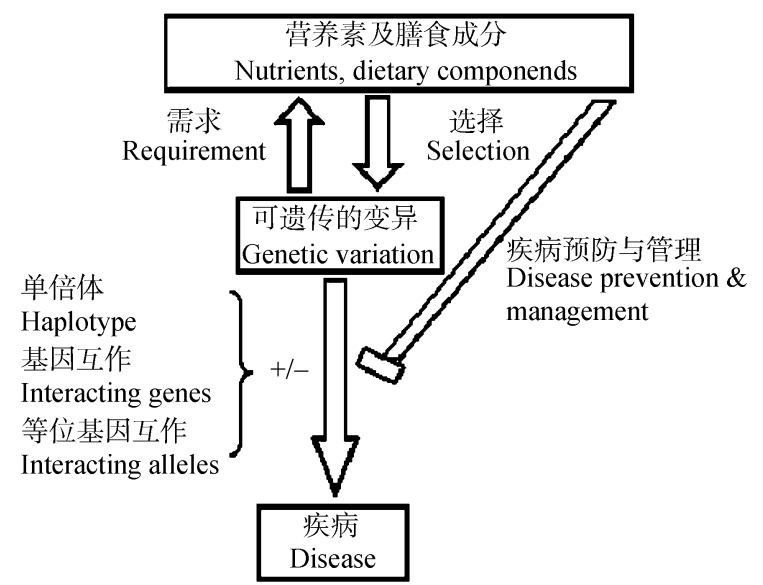

图 1 营养基因组学示意图

Fig. 1 Schema of nutrigenomics

基因组学技术可以帮助确认一些与疾病发生有 关的基因, 从而建立个性化食谱, 使人们的健康状 况通过调整饮食来达到最佳。它不仅可以了解食品 活性成分对人体代谢途径及体内平衡影响外，还可 以了解食品功能成分对不同人体基因多态性敏感的 差异, 并由此来调节饮食, 制定最合适的个性化膳 食, 并可有效地防止人体内与疾病相关基因的表 达。其主要研究内容包括以下方面：了解食物活性 成分如何直接或间接地影响人体内基因组结构的变 化; 探讨膳食因子可营养素对人体基因组产生的影 响; 探讨哪些慢性或遗传性疾病容易受到膳食因子 的影响；依据人体基因多态性的差异，探讨健康人 体和疾病患者对不同膳食因子敏感性的差异; 根据 不同人对营养需求、状态及其自身基因多态性的差 异来设计个性化膳食, 藉此达到预防慢性疾病的发 生 ${ }^{[9]}$ 。

许多营养素通过转录系统选择性的改变基因表 达，调节不同组织、不同环境条件下特定基因组的 活性。营养成分如氨基酸、脂肪酸和糖等, 都会影 响基因的表达，其作用方式可以是通过控制基因构 型或通过代谢产物 ${ }^{[10]}$ 或代谢状态(如激素状况、细胞 
氧化还原状况等等), 继而导致mRNA水平和(或)蛋 白质水平甚至其功能的改变。因此, 在营养研究中, 基因组学和蛋白质组学利用细胞培养、动物和人类 寻找和鉴定对某些营养素、药物或食物有良好反应 的特殊标志物。

同时, Jim和Rodriguez ${ }^{[11]}$ 认为, 在进行营养基因 组学研究时, 应注意以下 5 个方面: 产生适当的代 谢反应需要多少营养素, 特别是需要多少宏量营养 素; 对于遗传背景不同的人, 在复杂的膳食成分下 如何获得适量的营养素; 如何将膳食成分同机体代 谢的精细和长期调控联系起来; 在现有的分子和基 因组技术条件下，如何获得不同人自出生到死亡期 间的营养需要的变化量; 如何确保以一种对社会负 责的态度正确利用基因组学信息, 特别是当它与健 康状况不同的人群，如不同种族、贫富不一和未投 保的人。

\section{2 营养基因组学主要研究方法}

目前应用于营养基因组学研究的方法与功能基 因组学的研究相类似, 主要有 DNA 芯片技术、生物 标志物、蛋白质组学技术等。

\subsection{DNA 芯片技术 $[12]$}

DNA 芯片，又称基因芯片或微阵列 (microarrays)。其技术原理是基于DNA碱基的配对和 互补，把DNA或RNA分解为一系列碱基数固定交错 且重叠的寡核苷酸并进行测序，然后进行序列拼 接。主要流程包括待测基因的酶切成不同长度的片 段, 苂光定位标记, 然后与DNA芯片杂交, 应用激 光共聚焦苂光显微镜扫描芯片, 由于生物标记受激 光激发后发出苂光, 并且其强度与杂交程度有关, 可以获得杂交的程度和分布。根据探针的位置和序 列就可确定靶序列相应基因的序列或表达及突变情 况。该技术可以检测营养素对整个细胞、组织甚至 整个系统及作用方式上的差异 ${ }^{[13]}$ 。研究表明, 采用 高密度寡聚核酸微阵列通过比较成年小鼠和老年小 鼠gastocnemius muscle基因表达的总体变化，从而 发现与衰老相关的基因, 并进一步研究了能量限饲 对衰老的影响。Rao等人采用微阵列芯片对低硒日粮 的C57BL6 小鼠小肠的基因表达进行检测，与高硒 日粮组相比有 84 个基因的表达量增加两倍，而 48 个基因表达减少了 $75 \%$ ，其中高表达的主要与DNA 损伤，氧化诱导、细胞增殖等基因有关; 表达减少的
主要有谷胱甘肽过氧化物酶、P4503AI、2B9 等, 结 果表明硒含量可能调节与肿瘤形成有关的多个途 径 ${ }^{[14]}$ 。Lyakhovieh等检测了经 1,25-维生素D 处理过 的乳腺癌细胞的FGF-7 表达, 首次揭示了维生素D可 能过通调节FGF-7 的表达调节细胞的生长分化。

\section{2 生物学标记(biomarker)}

生物标志物(biomarker)通常是与疾病发生相关 的蛋白质, 在疾病的诊断、分级、预后及治疗监测 过程中常被作为诊断指标进行定量测定。基因组、 蛋白质组技术因为能在特定的条件下规模化地研究 基因和蛋白质的表达情况，所以为生物标志物的发 现、鉴定和评价提供了有力的技术平台。营养学家 通过人体干扰试验进行膳食营养研究, 在预防或促 进这一概念上许多慢性衰老疾病和失调都与营养有 关, 营养素参与疾病发生的初期预防, 相关的人体 干扰研究都用生物标记来确定营养素干扰的作用。 研究营养素对健康人体的后期作用需要采用新的生 物标记, 但目前还没有能够准确、专一、足够灵敏 的生物标记来确定其在疾病发作前的病理学变化。 将基因组学技术用于营养研究, 将许多小变化组合 成新的生物标记使生物标记变得非常灵敏, 可以做 到对病变的早期诊断。

\section{3 蛋白质组学技术}

\subsection{1 双向凝胶电泳 ${ }^{[15]}$}

其基本原理是第一向基于蛋白质等电点的不同 用等电聚焦分离, 第二向基于分子量的不同进行 SDS-PAGE 分离, 使蛋白质在二维平面上分开。翻 译后修饰和加工对蛋白质正常生理功能是必需的, 它们的变化往往与疾病有关。双向凝胶电泳中发现 的蛋白拖曳现象很可能使蛋白的不同翻译后修饰产 物所造成的拖曳图像的变化对营养素的研究提供了 帮助。人们在对大肠杆菌的研究中发现碳氮磷及硫 等元素缺乏会导致的细胞内蛋白质图谱变化, 而当 磷不足时, 发现有 137 个蛋白质的合成速率下降, 其中大部分表现为诱导合成, 其他则被抑制。

\subsection{2 质谱分析技术}

质谱 ${ }^{[16]}$ 已成为连接蛋白质与基因的重要技术, 是大规模自动化鉴定蛋白质的重要方法，因为蛋白 质的酶解是有规律可寻的, 每种蛋白酶针对特定蛋 白的酶解的片断质量和数量都较恒定。质谱分析能 精确地检测某种蛋白质经特定酶解后的质量和数量, 
与已建立好的蛋白数据库对照从而确定该蛋白的种 属。其优点是对待测检验物纯度要求不高, 可直接 对酶解液进行分析，具有灵敏度高速度快等特点。 用来分析蛋白质或多肽的质谱有两个主要部分：(1) 样品的离子源; (2)测量分子量的装置。一种是基质辅 助激光解吸附电离飞行时间质谱(MALDI-TOF)为一 脉冲式的离子化技术，它从固相标本中产生离子, 并在飞行管中测其分子量。另一种是电喷雾质谱 (ESI-MS), 是一连续离子化的方法, 从液相中产生 离子, 联合四极质谱或在飞行时间检测器中测其分 子量 ${ }^{[17]}$ 。近年来, 质谱的装置和技术有了长足的进 展, 在MALDI-TOF中, 最重要的改进是离子反射器 的延迟提取, 可达相当精确的分子量。在ESI-MS中, 纳米级电雾源的出现使微升级的样品在 30 40 min 内分析成为现实。

\section{3 营养基因组学的应用}

基因组营养学涵盖了一个广泛的领域，它研究 营养素和基因表达的相互影响 ${ }^{[18]}$ 。基因组营养学的 关键作用之一在于探讨特殊基因多态性和营养素个 体化反应之间的联系。随着对人类单核苷酸多态性 认识的不断深入, 其目标就是基于患者个体的基因 型特征，预测其对营养素的反应，以提供个性化膳 食营养建议，而非基于群体做出一般建议，最终达 到基因治疗的目的 ${ }^{[19]}$ 。营养基因组学的目的不是改 变用于消费的各种食品以提高它们的营养成分，而 是在于食用那些食品，如何让基因更好地适应，如 何改变基因的作用和结构。同时，如何根据每个人 自己的基因特点制定食谱，补充特定的营养成分， 以弥补由于基因变异造成对健康的影响。有的还可 以防止某些基因突变或改变基因的活动情况，从而 达到预防疾病、延缓衰老、促进健康的目的。

应用基因组学技术将阐明与营养相关的单核苷 酸多态性(SNPs), 并用来研究人体对某些疾病易感 性以及对营养素(食物)需求的个体间差异。营养素的 推荐膳食供给量或膡食参考摄入量都是对群体而言 的，然而人与人的基因是有差异的。人的基因组中 约有 140 万 200 万个SNPs, 其中 6 万多个存在于外 显子中，这可能是人体对营养素需求及响应差异的 重要分子基础[20]。因此, 将来的营养学研究将逐渐 趋向个体化，通过对基因构成以及代谢型的鉴定， 给出每个人的最佳食谱。

南加利福尼亚大学凯克医学院和洛杉矶加利福
尼亚大学、格劳医学院的研究表明 : 基因突变与动 脉硬化有关。研究指出：体内有 5-脂肪氧合酶 (5-lipoxygenase, ALOX5)突变体的人患动脉硬化的 风险较高。动脉硬化是指胆固醇在动脉壁上积聚而 导致心脏病。洛杉矶加利福尼亚大学动脉硬化研究 的参与者中有 $5 \%$ 体内出现这种 ALOX5 变体。这种 基因突变体会因摄入 $\omega-6$ 多不饱和脂肪酸而增强, 但摄入含 $\omega-3$ 多不饱和脂肪酸则可使其受到阻碍。 因此，对携带 ALOX5 突变体的人应少食含有 $\omega-6$ 多 不饱和脂肪酸的肉类和植物油，多食用富含 $\omega-3$ 不 饱和脂肪酸的鱼类如鲑鱼, 还应适当补充含二十碳 五烯酸(eicosapentaenoic acid，EPA)和二十二碳六烯 酸(docosahexaenoic acid, DHA)的深海鱼油制剂和维 生素 $\mathrm{E} 。$

叶酸代谢是近年来医学、遗传学和营养学研究 的热点之一, 主要是因为人们发现叶酸与神经管畸 形、肿瘤和心血管疾病有关 ${ }^{[21]}$ 。已知MTHFR是同型 半胱氨酸代谢中的关键酶之一，这种酶的活性和热 稳定性都较低。现已证实, 这种热不稳定性是由 MTHFR基因等 677 位的碱基由 $\mathrm{C} \rightarrow \mathrm{T} 、 1298 \mathrm{~A} \rightarrow \mathrm{C}$ 型 等的突变所造成的 ${ }^{[22]}$ 。MTHFR基因的遗传变异体在 叶酸水平较低时易造成中度的高半胱氨酸血症, 这 种遗传与营养的交互作用，增加了心血管疾病的神 经管缺陷的危险性。而实验和临床资料表明，大剂 量补充叶酸可以增加体内 5-甲基四氢叶酸生成, 从 而降低血浆同型半胱氨酸水平，减少心血管疾病的 发病和神经管畸形儿童的出生率。由此推断, 就 MTHFR基因突变的个体而言, 他们对叶酸的需求要 高于普通人群。

另一个SNP是如何改变基因表达的突出例子就 是乳糖耐受性例子。成年人一般都是乳糖不耐受的。 而产生这种现象的是 9000 年前的北欧人的乳糖分 解酶-根皮苷水解酶(lactase-phlorizin hydrolase, LCH) 基因发生的一个突变。尽管在这个基因中有 11 个 SNP，而这些SNP又可以分成 4 种(A，B，C和U)常见 的单倍体型, 在位于 $\mathrm{LCH}$ 上游 $14 \mathrm{~kb}$ 处 $\mathrm{C} 13910 \mathrm{~T}$ 的 SNP则与乳糖的而受性显著关联 [23]。通常认为这个 多突变可以改变蛋白质-DNA间的相互作用，从而 对基因的表达进行调控 ${ }^{[24]}$ 。被认为乳糖耐受性的 $\mathrm{A}$ 单倍体型在北欧人群中的分布频率为 $86 \%$, 而在南 欧人群中的频率却只有 $36 \%$ 。在群体中的这种优势 基因型对于营养水平的提高，预防脱水和改善钙的 吸收具有重要的作用。在其他启动子中的调控型 
SNPs (rSNPs)似乎对于基因的表达调控具有重要的 作用 ${ }^{[25 \sim 27]}$ 。

\section{4 展 望}

目前, 营养基因组学的研究正在不断的发展, 科学家们越来越不倾向于从性质或营养作用方面找 答案, 而是倾向于研究以营养基因组学为基础的系 统生物学的相互影响以促进健康。我们相信, 随着 有关各种族基因特点的巨大资料库的建立和记录人 类基因组信息的人类基因组芯片的出现，不仅为科 学家和医生们进行疾病研究而且也为促进人类健康 的基因营养提供依据,并将为营养基因学开拓更加 广阔的应用前景。

有专家预测, 营养基因组学将会是继药物基因 组学之后让世人瞩目的新的焦点学科。它不仅有助 于人们更好地理解个体由于基因差异而对各种食物 成分以及饮食方式所产生的不同反应，而且相关的 营养基因组数据也会为特定人群研制有效的食疗方 案打下扎实的基础。营养基因组学的未来发展有望 像药物基因组学打造“个性化药物”那样, 为人们量 身定做出能满足个体需求的“个性化食品”。在揭示 人类遗传密码顺序的人类基因组图谱绘制成功之后, 一项以基因组为基础的营养学研究将给疾病治疗带 来一场革命。那时，人们可以根据各自的基因图谱 制定一份个性化的饮食方案, 以此防病治病。

\section{参考文献(References):}

[1] Watson JD, Crick FHC. Molecular structure of nucleic acids: a structure for deoxyribose nucleic acid. Nature, 1953, 171(4356): 737-738.[DOI]

[2] Lander ES, Linton LM, Birren B, Nusbaum C, Zody MC, Baldwin J, Devon K, Dewar K, Doyle M, FitzHugh W, Funke R, Gage D, Harris K, Heaford A, Howland J, Kann L, Lehoczky J, LeVine R, McEwan P, McKernan K, Meldrim J, Mesirov JP, Miranda C, Morris W, Naylor J, Raymond C, Rosetti M, Santos R, Sheridan A, Sougnez C, Stange-Thomann N, Stojanovic N, Subramanian A, Wyman D, Rogers J, Sulston J, Ainscough R, Beck S, Bentley D, Burton J, Clee C, Carter N, Coulson A, Deadman R, Deloukas P, Dunham A, Dunham I, Durbin R, French L, Grafham D, Gregory S, Hubbard T, Humphray S, Hunt A, Jones M, Lloyd C, McMurray A, Matthews L, Mercer S, Milne S, Mullikin JC, Mungall A, Plumb R, Ross M, Shownkeen R, Sims S, Waterston RH, Wilson RK, Hillier LW, McPherson JD, Marra MA, Mardis ER, Fulton LA, Chinwalla AT, Pepin KH, Gish WR, Chissoe SL, Wendl MC, Delehaunty KD, Miner TL, Delehaunty A,
Kramer JB, Cook LL, Fulton RS, Johnson DL, Minx PJ, Clifton SW, Hawkins T, Branscomb E, Predki P, Richardson P, Wenning S, Slezak T, Doggett N, Cheng JF, Olsen A, Lucas S, Elkin C, Uberbacher E, Frazier M, Gibbs RA, Muzny DM, Scherer SE, Bouck JB, Sodergren EJ, Worley KC, Rives CM, Gorrell JH, Metzker ML, Naylor SL, Kucherlapati RS, Nelson DL, Weinstock GM, Sakaki Y, Fujiyama A, Hattori M, Yada T, Toyoda A, Itoh T, Kawagoe C, Watanabe H, Totoki Y, Taylor T, Weissenbach J, Heilig R, Saurin W, Artiguenave F, Brottier P, Bruls T, Pelletier E, Robert C, Wincker P, Smith DR, Doucette-Stamm L, Rubenfield M, Weinstock K, Lee HM, Dubois J, Rosenthal A, Platzer M, Nyakatura G, Taudien S, Rump A, Yang H, Yu J, Wang J, Huang G, Gu J, Hood L, Rowen L, Madan A, Qin S, Davis RW, Federspiel NA, Abola AP, Proctor MJ, Myers RM, Schmutz J, Dickson M, Grimwood J, Cox DR, Olson MV, Kaul R, Raymond C, Shimizu N, Kawasaki K, Minoshima S, Evans GA, Athanasiou M, Schultz R, Roe BA, Chen F, Pan H, Ramser J, Lehrach H, Reinhardt R, McCombie WR, de la Bastide M, Dedhia N, Blöcker H, Hornischer K, Nordsiek G, Agarwala R, Aravind L, Bailey JA, Bateman A, Batzoglou S, Birney E, Bork P, Brown DG, Burge CB, Cerutti L, Chen HC, Church D, Clamp M, Copley RR, Doerks T, Eddy SR, Eichler EE, Furey TS, Galagan J, Gilbert JG, Harmon C, Hayashizaki Y, Haussler D, Hermjakob H, Hokamp K, Jang W, Johnson LS, Jones TA, Kasif S, Kaspryzk A, Kennedy S, Kent WJ, Kitts P, Koonin EV, Korf I, Kulp D, Lancet D, Lowe TM, McLysaght A, Mikkelsen T, Moran JV, Mulder N, Pollara VJ, Ponting CP, Schuler G, Schultz J, Slater G, Smit AF, Stupka E, Szustakowski J, Thierry-Mieg D, Thierry-Mieg J, Wagner L, Wallis J, Wheeler R, Williams A, Wolf YI, Wolfe KH, Yang SP, Yeh RF, Collins F, Guyer MS, Peterson J, Felsenfeld A, Wetterstrand KA, Patrinos A, Morgan MJ, de Jong P, Catanese JJ, Osoegawa K, Shizuya $\mathrm{H}$, Choi S, Chen YJ. Initial sequencing and analysis of the human genome. Nature, 2001, 409(6822): 860-921.[DOI]

[3] Vay Liang WG, Ritva RB, Debra AW. Diet, nutrition, cancer prevention: the postgenomic era. J Nutr, 2003, 133(11): 3830S-3836S.

[4] Tefferi A. Genomics basics: DNA structure, gene expression, cloning, genetic mapping, and molecular tests. Seminars in Cardiothoracic and Vascular Anesthesia, 2006, 10(4), 282-290.[DOI]

[5] Walker WA, Blackburn G. Symposium introduction: nutrition and gene regulation. J Nutr, 2004, 134(9): 2434S-2436S.

[6] Ruan E, Teng TO. Science, medicine, and the future: nutritional genomics. BMJ, 2002, 324(6): 1438-1442.[DOI]

[7] Payne PW Jr, Royal C, Kardia SL. Genetic and social environment interactions and their impact on health policy. J Am Acad Orthop Surg, 2007, 15(1): S95-S98. 
[8] Patrick J. Stover. Nutritional genomics. Physiol Genomics, 2004, 16(2): 161-165.

[9] Schork NJ, Fallin D, Lanchbury JS. Single nucleotide polymorphisms and the future of genetic epidemiology. Clin Gene, 2000, 58(4): 250-264.[DOI]

[10] Michael JG, Marianne W, Lorraine B, Helen MR, Bruce G, Ben VO. Metabolomics in human nutrition: opportunities and challenges. Am J Clin Nutr, 2005, 82(3): 497-503.

[11] Jim K, Rodriguez RL. Nutritional genomics: the next frontier in the postgenomics era. Physiol Genomics, 2004, 16(8): 166-177.

[12] Schumacher A, Kapranov P, Kaminsky Z, Flanagan J, Assadzadeh A, Yau P, Virtanen C, Winegarden N, Cheng J, Gingeras T, Petronis A. Microarray-based DNA methylation profiling: technology and applications. Nucleic Acids Res, 2006, 34(2): 528-542.[DOI]

[13] Kato H, Kimura T. Evaluation of the dietary intake of proteins and amino acids by DNA microarrays technology. J Nutr, 2003, 133(6): 2073S-2077S.

[14] Rao L, Puschner B, Prolla TA. An expression profiling of low selenium slatus in the mouse intestine, transcriptional activation of genes linked to DNA damage, cell cycle control and oxidative stress. J Nutr, 2001, 131(12): 3175-3181.

[15] Wang J, Li D, Dangott LJ, Wu G. Proteomics and its role in nutrition research. J Nutr, 2006, 136(7): 1759-1762.

[16] Ross SA, Srinivas PR, Clifford AJ, Lee SC, Philbert MA, Hettich RL. New technologies for nutrition research. $J$ Nutr, 2004, 134(3): 681-685.

[17] Kim H, Page GP, Barner S. Proteomics and mass spectrometry in nutrition research. J Nutr, 2004, 20(1): 1 $55-165$.

[18] Walker WA, Blackburn G. Nutrition and gene regulation. J Nutr, 2004, 134(9): 2434S-2436S.

[19] Vay Liang WG, Christine THN, Harris DM, Lee WN. Nutrient-gene interaction: metabolic ge- notype-phenotype. J Nutr, 2005, 135(12): 3016S-3020S.

[20] Sachidanandam R, Weissman D, Schmidt SC, Kakol JM,
Stein LD, Marth G, Sherry S, Mullikin JC, Mortimore BJ, Willey DL, Hunt SE, Cole CG, Coggill PC, Rice CM, Ning Z, Rogers J, Bentley DR, Kwok PY, Mardis ER, Yeh RT, Schultz B, Cook L, Davenport R, Dante M, Fulton L, Hillier L, Waterston RH, McPherson JD, Gilman B, Schaffner S, Van Etten WJ, Reich D, Higgins J, Daly MJ, Blumenstiel B, Baldwin J, Stange-Thomann N, Zody MC, Linton L, Lander ES, Altshuler D. A map of human genome sequence-variation containing 1.42 million single nucleotide polymorphisms. Nature, 2001, 409 (6822): 928-933.[DOI]

[21] Lathrop SL, Shane B, Bagley PJ. Combined folate and riboflavin status affect homocysteine methylation in cultured immortalized lymphocytes from homozygous for the MTHFR C667T mutation. $J$ Nutr, 2003, 133(1): 2716-2720.

[22] Milner JA. Molecular targets for bioactive food components. J Nutr, 2004, 134(9): 2492S-2498S.

[23] Enattah NS, Sahi T, Savilahti E, Terwilliger JD, Peltonen L, Jarvela I. Identification of a variant associated with adult-type hypolactasia. Nat Genet, 2002, 30(2): 233-237.[DOI]

[24] Hollox EJ, Poulter M, Wang Y, Krause A, Swallow DM. Common polymorphism in a highly variable region upstream of the human lactase gene affects DNA-protein in teractions. Eur J Hum Genet, 1999, 7(7): 791-800. [DOI]

[25] Benbow U, Tower GB, Wyatt CA. High levels of MMP-1 expression in the absence of the $2 \mathrm{G}$ single nucleotide polymorphism is mediated by $\mathrm{p} 38$ and ERK1/2 mitogenactivated protein kinases in VMM5 melanoma cells. $J$ Cell Biochem, 2002, 86(2): 307-319.[DOI]

[26] Bream JH, Ping A, Zhang X, Winkler C, Young HA. A single nucleotide polymorphism in the proximal IFN-gamma promoter alters control of gene transcription. Genes Immun, 2002, 3(3): 165-169.[DOI]

[27] Lamba JK, Lin YS, Schuetz EG, Thummel KE. Genetic contribution to variable human CYP3A- mediated metabolism. Adv Drug Deliv Rev, 2002, 54(10): 1271-1294.[DOI] 